Ann. Généi. Sél. anim., I970, 2 (3), 249-26r.

\title{
HÉRÉDITÉ DES PENDELOQUES DANS LA RACE OVINE SARDE
}

\author{
S. CASU, J. G. BOYAZOGLU et J.-J. LAUVERGNE \\ Istituto Zootecnico e caseario per la Sardegna, via Oriani 4, Sassari \\ Sardegna, Italia \\ Animal Husbandry and Dairy Research Institute, Pretoria \\ South Africa \\ Siation centrale de Génétique animale, \\ Centre national de Recherches zootechniques, 78-Jouy-en-Josas \\ Institut national de la Recherche agronomique \\ France
}

\section{RÉSUMÉ}

Dans la race de brebis laitières sarde en Sardaigne, on observe un certain nombre d'animaux des deux sexes qui présentent généralement deux, parfois une seule pendeloque au dessous du cou. On note une certaine variation dans la forme de ces appendices. Comme chez la chèvre et comme dans d'autres races de moutons géographiquement très éloignées de la Sardaigne (brebis valaques ou Navajo), le caractère présence de pendeloques semble déterminé par un gène autosomal dominant $(W)$ à pénétrance totale ou subtotale. Ce gène aurait une certaine influence sur la reproduction et la production laitière des femelles tout au moins, les mâles n'ayant pu être étudiés. Les brebis munies de pendeloques ( $W W$ ou $W w$, ou les deux) se révèlent supérieures pour ces deux caractères aux animaux sans pendeloques $w w$.

L'examen de la fréquence génique de $w$ à travers 25 troupeaux de sélection $\left(\bar{q}^{*}=0,847\right)$ n'infirme pas l'hypothèse d'équilibre génique. Dans ce cas, on devrait supposer qu'il existe une overdominance, le génotype $W w$ étant, chez les femelles tout au moins, supérieur aux deux homozygotes. Des études comparées directes sont nécessaires pour préciser ce point de même qu'il faudra analyser en détail le phénomène de migration des mâles à travers les troupeaux et ceux de l'ancienneté et de l'intensité de la sélection qui a pu favoriser tel ou tel génotype pour savoir si l'équilibre génique est réalisé au niveau de l'ensemble de la population de l'lle.

\section{INTRODUCTION}

L'existence des pendeloques (I) chez les Ongulés domestiques (Chèvre, Mouton, Porc) et sauvages (Cerf, Sanglier) est connue depuis fort longtemps, elle a déjà été décrite par Goubaux (I852) chez la Chèvre et le Mouton, et Blanc (I897) chez la Chèvre et le Porc.

(1) Plusieurs autres noms ont été donnés à ces appendices : breloques, sonnettes, barbes, pendants, glandes, etc. En Italie, les noms " lacinie " et " pendenti " prédominent, bien que dans certaines régions les bergers les appellent autrement (" naccaras ", "bargigli " et aussi " campaneddas "). En anglais, on dit " wattles". 
Chez le Mouton, une note statistique sur la fréquence des pendeloques a déjà été publiée par MouQuet (I895). VASIN (I928), en croisant des brebis valaques à " barbes " avec un bélier Mérinos dépourvu de ces appendices et en faisant le croisement de retour, concluait à l'existence d'un gène dominant simple $(W)$. Bı,UNN (I943) rapportait aussi que le mode de transmission héréditaire de ce caractère dans une population de moutons Navajo était dû̀ ù gène dominant simple (cf. SERRA, I948, pour revue). Cette hypothèse a également été vérifiée chez la Chèvre domestique (Lush, I926; RICORDEAU, I967).

En Sardaigne, où l'on élève environ 2500000 brebis laitières de race sarde à peu près également réparties dans les trois provinces de Sassari, Nuoro et Cagliari, ce caractère se rencontre fréquemment.

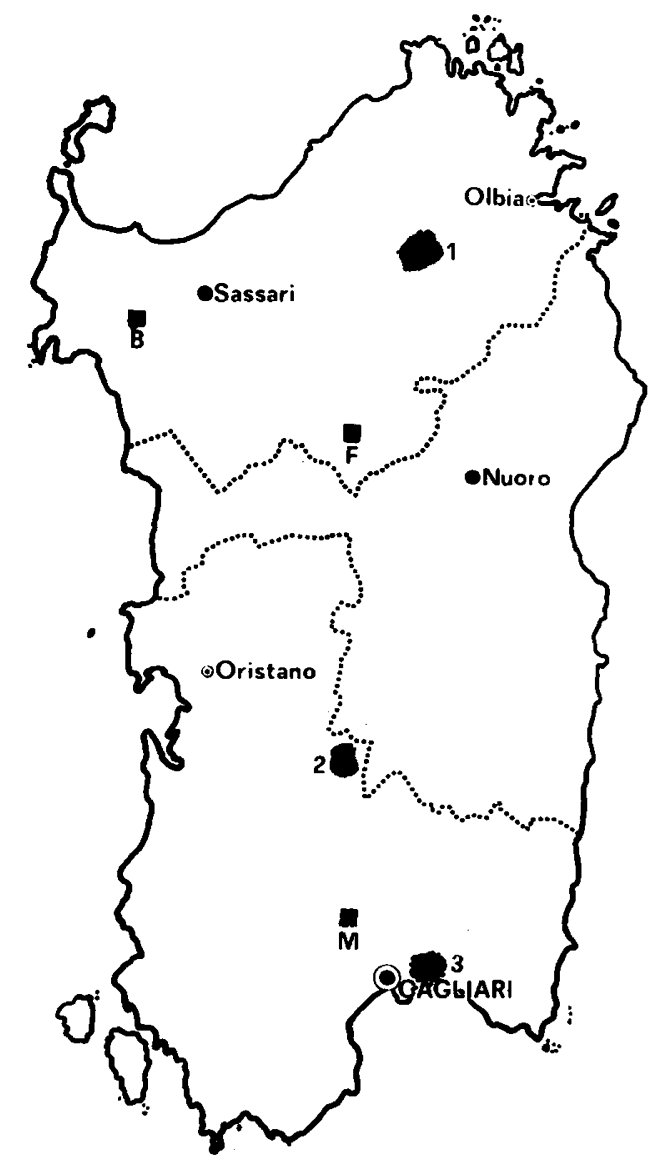

FIG. I. - Localisation en Sardaigne des 3 troupeaux expérimentaux (B = Bonasai, $\mathrm{F}=$ Foresta di Burgos, $\mathrm{M}=$ Monastir) et des 3 régions d'élevage, ( $\mathrm{I}$ dans la province de Sassari, 2 et 3 dans la province de Cagliari).

Les données dont nous disposons pour la présente étude proviennent d'observations faites dans les trois troupeaux expérimentaux de l'i Istituto Zootecnico e Caseario per la Sardegna " (Bonassai, Foresta di Burgos et Monastir), et dans 
25 troupeaux privés de trois régions d'élevage ( $\mathrm{I}, 2$ et 3) de Sardaigne (fig. I). Nous verrons successivement le déterminisme héréditaire du caractère, l'influence du ou des facteurs génétiques sur les caractères de production et la génétique de population.

\section{I. - LE DÉTERMINISME HÉRÉDITAIRE}

\section{A. - Matériel et Méthodes}

Nous avons utilisé les observations portant sur les descendants de femelles avec ou sans pendeloques originaires des troupeaux expérimentaux en 1968 et 1969 accouplées à 10 béliers porteurs de pendeloques et à 31 béliers sans pendeloques. Dans ces troupeaux, comme dans les autres troupeaux de l'Institut, la présence des pendeloques est régulièrement contrôlée à la naissance pour les agneaux et leurs mères, puis, pour les agnelles, au sevrage, entre deux et trois mois et entre six et sept mois. Étant abattus peu après la naissance, la plupart des mâles ne peuvent être examinés qu'une seule fois.

Une grande variabilité a été observée dans la position, la forme et les dimensions des pendeloques. En ce qui concerne le nombre (par animal) on a observé quelques animaux possédant une seule pendeloque, mais moins fréquemment que chez la Chèvre (RIcordeau, 1967) : 11 cas avec une seule pendeloque sur 771 animaux considérés. D'autre part, une différence de dimensions et de forme a été fréquemment trouvée entre les deux pendeloques du même animal (fig. 2).

Pour l'étude génétique factorielle, nous nous sommes contentés de noter la présence ou l'absence de pendeloques (une ou deux), ce qui ne peut prêter à contestation que pour les agneaux mâles qui, dans les conditions d'élevage évoquếes ci-dessus, doivent être examinés si sommairement que des imprécisions sont possibles.

\section{B. - Résultats et Discussion}

Dans un premier temps, on a examiné les deux accouplements qui permettent une interprétation factorielle simple (tabl. I) sur les descendants femelles seulement. Si l'on admet que les nombreux descendants mâles de type indéterminé

TABLEAU I

Résultats d'accouplèments permettant de formuler une hypothèse sur le déterminisme héréditaire de la présence des pendeloques dans la race sarde (troupeaux expérimentaux 1968 et 1969 , femelles seulement)

\begin{tabular}{|c|c|c|c|c|}
\hline \multicolumn{2}{|c|}{ Phénotype des parents } & \multicolumn{2}{|c|}{ Descendants femelles observés } & \multirow{2}{*}{$\begin{array}{c}\text { Fréquence } \\
\text { des descendants } \\
\text { sans pendeloques } \\
(\text { en \%) }\end{array}$} \\
\hline Pères & Mères & Avec pendeloques & Sans pendeloques & \\
\hline $\begin{array}{l}\text { avec } \\
\text { pendeloques }\end{array}$ & $\begin{array}{l}\text { sans } \\
\text { pendeloques }\end{array}$ & $13^{8}$ & 142 & 50,7 \\
\hline $\begin{array}{c}\text { sans } \\
\text { pendeloques }\end{array}$ & $\begin{array}{c}\text { sans } \\
\text { pendeloques }\end{array}$ & o & $66 I$ & I 00,0 \\
\hline
\end{tabular}





FIG. 2. - Variation dans le nombre et la forme des pendeloques en race ovine sarde A. Une seule pendeloque arrondie de taille moyenne.

B. Deux pendeloques dont une très petite et une seconde de taille moyenne, arrondie.

C. Deux pendeloques, une de taille moyenne plutôt allongée et une très grande pendante et arrondie.

D. Deux pendeloques très semblables, de taille moyenne, arrondies avec un court pédoncule.

E. Deux pendeloques très semblables, de grande taille et de type allongé.

F. Deux pendeloques très semblables de grande taille et de type arrondi. 
à la naissance ( $16 \%$ ) sont munis de pendeloques, on retrouve dans ce sexe des résultats comparables à ceux des femelles pour le premier accouplement. Pour le second, les résultats s'accordent bien à ceux des femelles $(509$ descendants mâles tous démunis de pendeloques).

Comme tous les béliers pourvus de pendeloques avaient donné dans leur descendance aussi bien des animaux avec pendeloques que sans pendeloques, on peut admettre qu'ils étaient tous hétérozygotes et 1'hypothèse d'un gène autosomal dominant responsable de la présence des pendeloques dans les deux sexes paraît donc valable. La pénétrance semble, à première vue, totale ou subtotale.

En reprenant après VASIN (I928) et RICORDEAU (I967) la notation de LusH (r926) chez la Chèvre, nous pouvons, tout au moins provisoirement, appeler $W$ ce gène dominant; w étant l'allèle " normal ".

Pour vérifier cette hypothèse sur la deuxième série de résultats que l'on a de ces mêmes troupeaux (mâles homo- ou hétérozygotes croisés à des femelles phénotypiquement munies de pendeloques : $W W$ ou $W w)$ on doit partir d'une valeur de $q$ (fréquence de $w$ ) qui est calculable si l'on connaît (ou si l'on suppose connu) le mode d'accouplement pratiqué dans ces troupeaux. Comme jusqu'à présent on n'a tenu aucun compte, au moins consciemment, du phénotype " pendeloque " pour le choix des reproducteurs et leur appariement, on peut penser que pour les gènes qui contrôlent ce caractère, il y a panmixie. Dans ces conditions, on peut admettre, en supposant en outre que l'on est au voisinage de l'équilibre génique, que les fréquences génotypiques sont proportionnelles aux termes du développement

TABLEAU 2

Résultats d'accouplements dans lesquels le génotype des femelles n'est pas exactement connu et où on a déterminé les fréquences géniques par le calcul en supposant réalisés la panmixie et l'équilibre génique

\begin{tabular}{|c|c|c|c|c|c|c|c|}
\hline \multicolumn{2}{|c|}{$\begin{array}{c}\text { Génotype } \\
\text { des parents }\end{array}$} & \multicolumn{3}{|c|}{ Descendants femelles observés } & \multicolumn{2}{|c|}{ Effectifs théoriques } & \\
\hline pères & $\begin{array}{l}\text { mères } \\
\text { avec } \\
\text { pende- } \\
\text { loques }\end{array}$ & $\begin{array}{c}\text { avec } \\
\text { pendeloques }\end{array}$ & $\begin{array}{c}\text { sans } \\
\text { pendeloques }\end{array}$ & total & $\begin{array}{c}\text { avec } \\
\text { pendeloques }\end{array}$ & $\begin{array}{c}\text { sans } \\
\text { pendeloques }\end{array}$ & $\chi^{2}$ \\
\hline$W w$ & $\begin{array}{l}W W \\
W w\end{array}$ & 48 & 19 & 67 & $5 \mathrm{I}, 25$ & r 5,75 & $\begin{array}{c}0,876 \\
\text { NS }\end{array}$ \\
\hline$\tilde{w} \omega$ & $\begin{array}{l}W W \\
W_{w}\end{array}$ & I IO & 92 & 202 & 107,0 & 95,0 & $\begin{array}{c}0,179 \\
\text { NS }\end{array}$ \\
\hline
\end{tabular}


de $(p+q)^{2}$. L'estimation $q^{*}$ de $q$ est alors $q^{*}=\sqrt{\frac{d}{n}}(d$ étant le nombre des homozygotes récessifs, $n$, l'effectif de l'échantillon) avec, pour écart-type, $s^{\prime}=\sqrt{\frac{4 n}{\mathrm{I}-q^{2}}}$, CotTERmaN (I964). Ce qui donne, avec les données recueillies en I968 et I969 (tabl. 5):

$$
q^{*}=0,888 ; \quad s^{\prime}=0,0056
$$

A partir de cette estimée nous avons déduit les fréquences attendues dans les 2 types de croisements du tableau 2 , et nous les avons comparées aux fréquences observées (descendance femelle uniquement). L'hypothèse d'un gène dominant autosomal semble parfaitement acceptable.

\section{II. - CARACTÉRISTIQUES DE REPRODUCTION}

ET DE PRODUCTION LAITIĖRE DES DIFFÉRENTS GÉNOTYPES

\section{A. - Matériel et Méthodes}

En 1969, dans les troupeaux expérimentaux de Bonassai et de Foresta di Burgos, on a entrepris une évaluation de la prolificité selon les phénotypes. On a pu réunir également des données de production laitière dans le troupeau de Bonassai sur des brebis de $1^{\mathrm{re}}, 2^{\mathrm{e}}, 3^{\mathrm{e}}$ et suivantes lactations.

\section{B. - Résultats}

Les données de prolificité sont rassemblées dans le tableau 3, celles de productions laitières dans le tableau 4 .

\section{C. - Discussion}

Les données des tableaux 3 et 4 indiquent une certaine supériorité des brebis munies de pendeloques ( $W W$ ou $W w$ ) sur celles qui n'en ont pas (ww), tant pour la prolificité que pour la production laitière. Pour la prolificité, ce résultat concorde avec les observations de Ricordeau (I967) sur la chèvre Saanen où les écarts étaient même plus grands. Il reste que, n'ayant pas distingué les deux génotypes $W W$ et $W w$ parmi les brebis avec pendeloques, on ne sait celui qui se classe en tête.

\section{III. - GÉNÉTIQUE DES POPULATIONS}

La supériorité que nous venons de détecter tant au point de vue prolificité que production laitière des brebis munies de pendeloques sur celles qui n'en ont pas, confère au moins à l'un des deux génotypes $W W$ ou $W w$, un avantage sélectif 


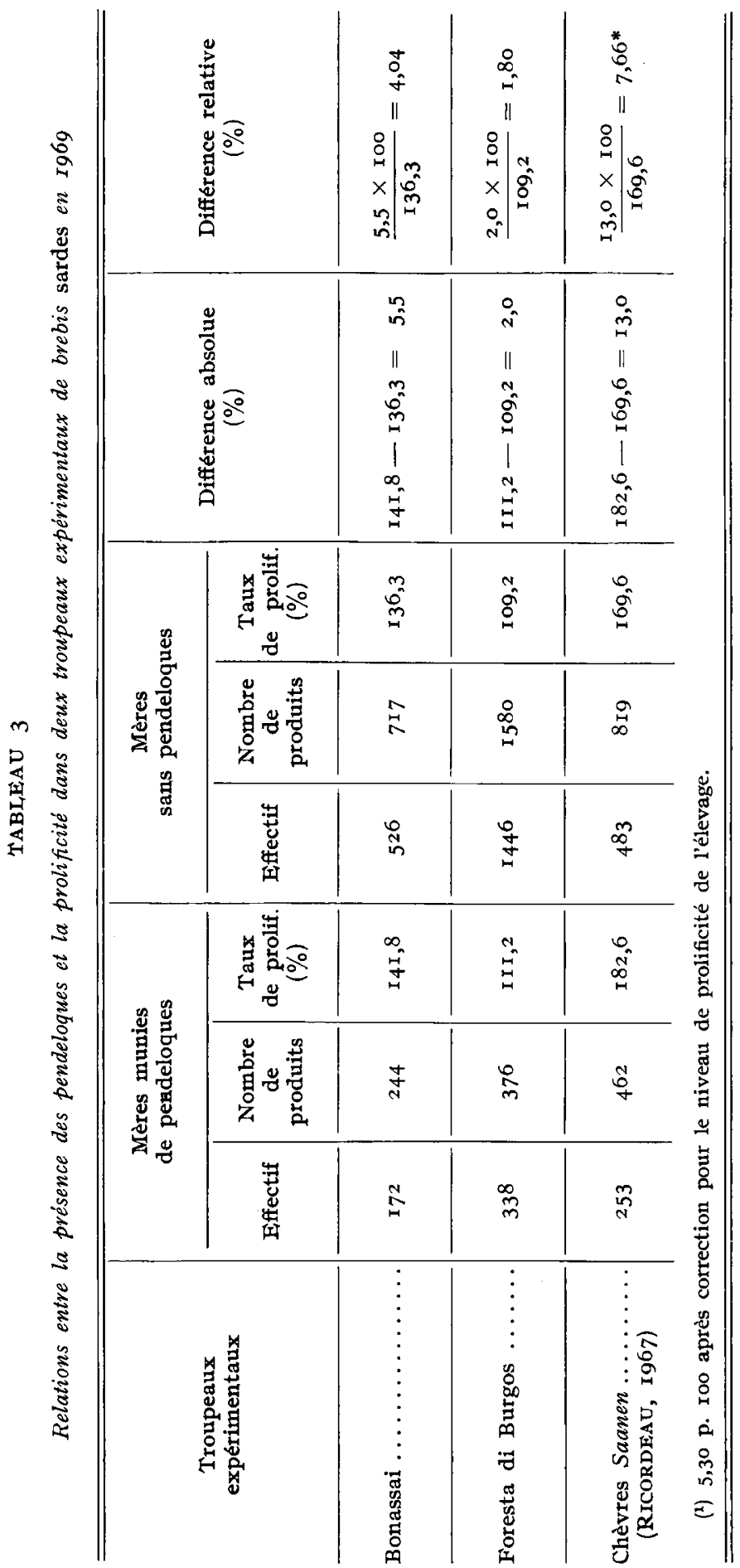




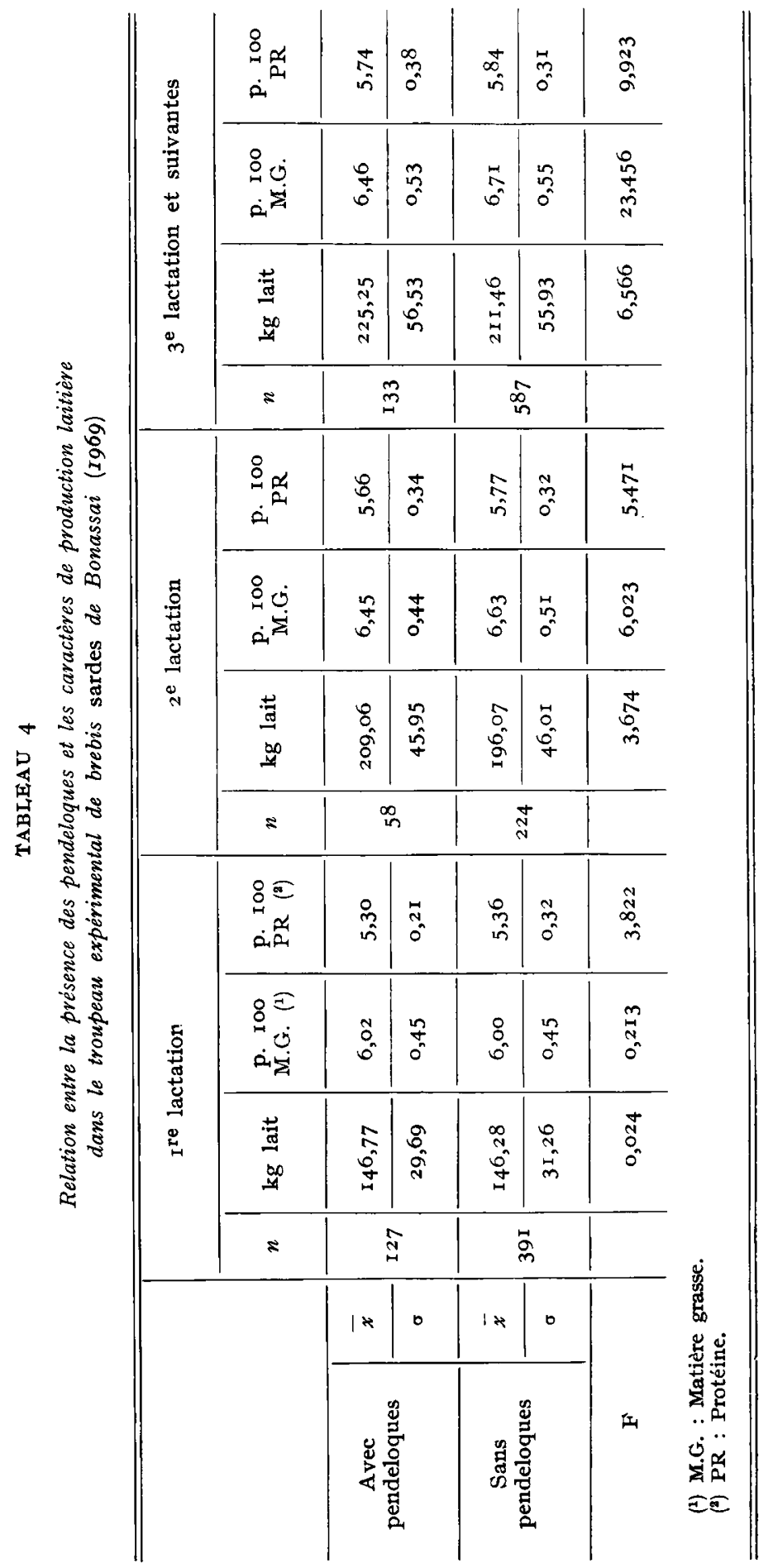


sur les homozygotes sans pendeloques (ww). Selon la valeur prise par les différents coefficients de sélection, on aboutira ou non à un équilibre de la fréquence génique. L'examen de cette dernière à travers les troupeaux de l'île dont nous avons les données s'impose donc tout d'abord. Ces données sont toutefois encore peu nombreuses et concernent uniquement les femelles.

\section{A. - Tableau de la fréquence $\mathrm{q} d u$ gène $\mathrm{w}$ (absence de pendeloques) à travers la population des brebis sardes}

\section{Matériel et Méthodes}

Nous disposons de deux groupes de données :

- des enregistrements de pendeloques faits dans les populations femelles des trois troupeaux de l'Institut (B, F, et $M$ de la carte de la fig. 1) soit 2394 observations en 1967, 2251 en 1968 et 2298 en 1969;

- des observations effectuées en 1969 dans 25 troupeaux de sélection répartis dans trois régions de l'lle géographiquement très éloignées $(1,2$ et 3 de la carte fig. 1) représentant au total 2587 observations :

- 4 troupeaux avec 578 observations dans la région 1, près d'Olbia (Nord de l'íle);

- 13 troupeaux avec 1041 observations dans la région 2 (centre sud de l'ile);

- 9 troupeaux avec 968 observations dans la région 3 , près de Cagliari (Sud de l'ile).

Moins précis que dans les troupeaux de l'Institut, les contrôles dans les troupeaux privés ont été effectués par des inspecteurs de l'Institut pour tous les animaux femelles adultes. On a noté la présence et le nombre des pendeloques.

Les calculs de $q^{*}$ estimée de $q$ et de son écart-type $s^{\prime}$ se font comme précédemment indiqué à partir des formules de CotTerman (1964).

\section{Résultats}

Les comptages dans les troupeaux expérimentaux pour les trois années I967, 68 et 69 sont donnés dans le tableau 5. Dans le tableau 6 nous avons présenté

TABLEAU 5

Estimées $\mathrm{q}^{*}$ de la fréquence du gène $\mathrm{w}$ chez les femelles des troupeaux expérimentaux, trois années successives

\begin{tabular}{|c|c|c|c|c|}
\hline $\begin{array}{l}\text { Troupeaux } \\
\text { et années }\end{array}$ & $\begin{array}{c}\text { Nombre } \\
\text { de brebis } \\
\text { sans pendeloques }\end{array}$ & $\begin{array}{l}\text { Taille } \\
\text { du troupeau } \\
n\end{array}$ & $\begin{array}{c}\text { Estimée } \\
\text { de la fréquence } \\
\text { de } w \\
q^{*}\end{array}$ & $\begin{array}{c}\text { Écart-type } \\
\text { de } q^{*} \\
s^{\prime}\end{array}$ \\
\hline 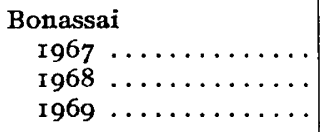 & $\begin{array}{l}8 \mathrm{I} 2 \\
723 \\
592\end{array}$ & $\begin{array}{r}1125 \\
956 \\
783\end{array}$ & $\begin{array}{l}0,850 \\
0,870 \\
0,870\end{array}$ & $\begin{array}{l}0,0079 \\
0,0080 \\
0,0088\end{array}$ \\
\hline $\begin{array}{l}\text { Foresta } \text { di Burgos } \\
\quad \text { r } 967 \ldots \ldots \ldots \ldots \ldots \ldots \\
\quad \text { r } 968 \ldots \ldots \ldots \ldots \ldots \\
\text { r } 969 \ldots \ldots \ldots \ldots \ldots\end{array}$ & $\begin{array}{l}637 \\
655 \\
724\end{array}$ & $\begin{array}{l}784 \\
800 \\
887\end{array}$ & $\begin{array}{l}0,902 \\
0,905 \\
0,904\end{array}$ & $\begin{array}{l}0,0077 \\
0,0075 \\
0,0072\end{array}$ \\
\hline $\begin{array}{l}\text { Monastir } \\
\text { r967 } \ldots \ldots \ldots \ldots \ldots \ldots \\
\quad \text { r968 } \ldots \ldots \ldots \ldots \ldots \\
\text { r969 } \ldots \ldots \ldots \ldots \ldots \ldots\end{array}$ & $\begin{array}{l}455 \\
442 \\
56 r\end{array}$ & $\begin{array}{l}485 \\
495 \\
628\end{array}$ & $\begin{array}{l}0,969 \\
0,945 \\
0,945\end{array}$ & $\begin{array}{l}0,0056 \\
0,0074 \\
0,0065\end{array}$ \\
\hline
\end{tabular}


TABLEAU 6

Estimées $\mathrm{q}^{*}$ de la fréquence $d u$ gène $\mathrm{w}$ chez les brebis de troupeaux sardes en 1969

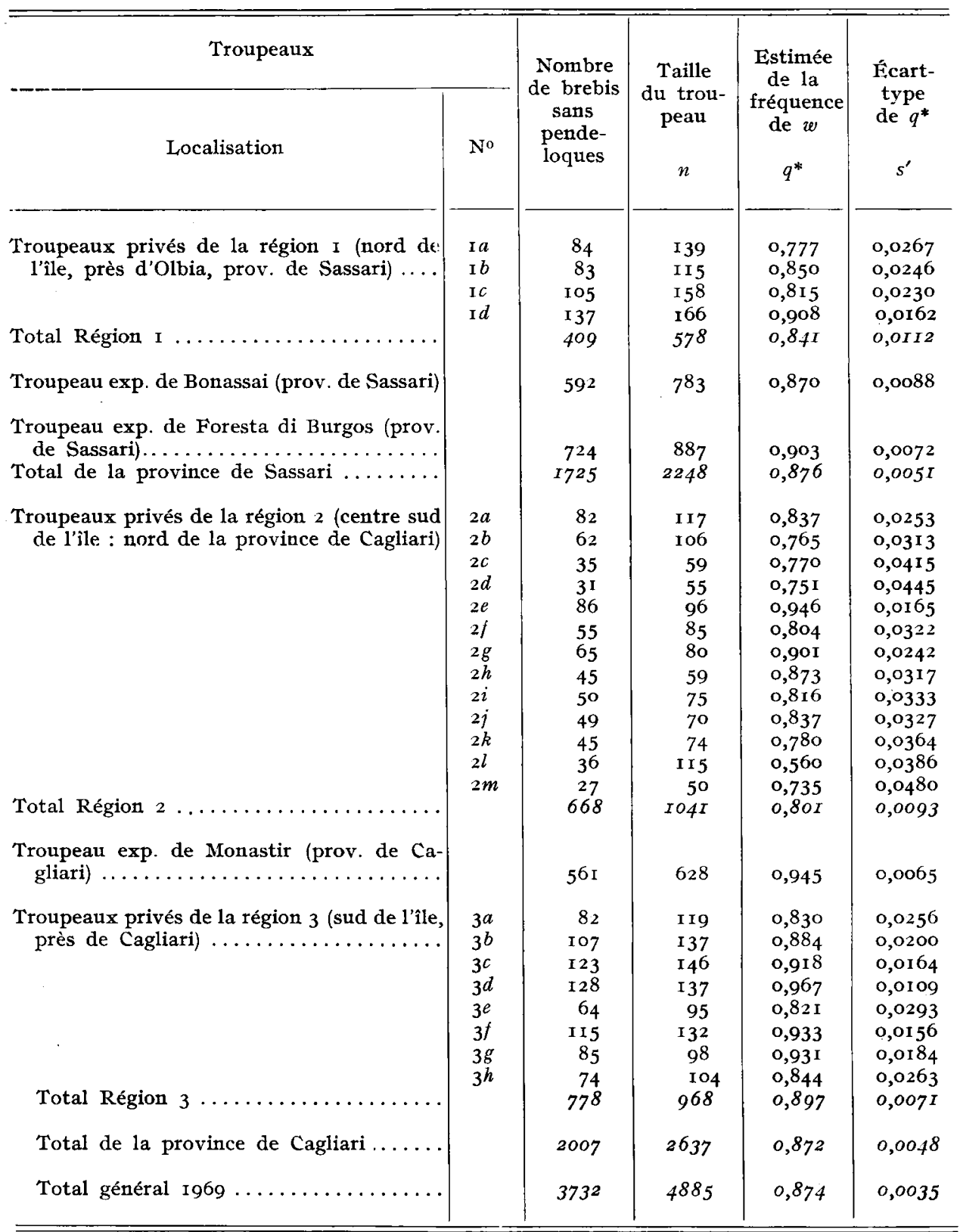

l'estimée de la fréquence génique dans les troupeaux privés et expérimentaux en I969 en allant du Nord au Sud de l'île. 
L'examen des fréquences à travers toute l'île révèle des différences significatives pour la fréquence entre les troupeaux et les régions mais, à une exception près, toutes ces fréquences se regroupent entre 0,75 et $0,95^{\circ}$ en comptant tous les troupeaux même les plus petits (tabl. 6). Si l'on dresse l'histogramme des fréquences géniques observées parmi les 25 troupeaux privés du tableau 6 , on note (fig. 3) que la répartition est très régulière autour de la moyenne $\left(\bar{q}^{*}=0,847\right)$.

\section{Discussion}

Pour affirmer que la distribution de la figure 3 est une distribution stationnaire des fréquences du gène $w$ parmi les isolats que constituent les troupeaux sardes, l'équilibre à l'échelon de la population de l'île étant atteint, il faudrait que les

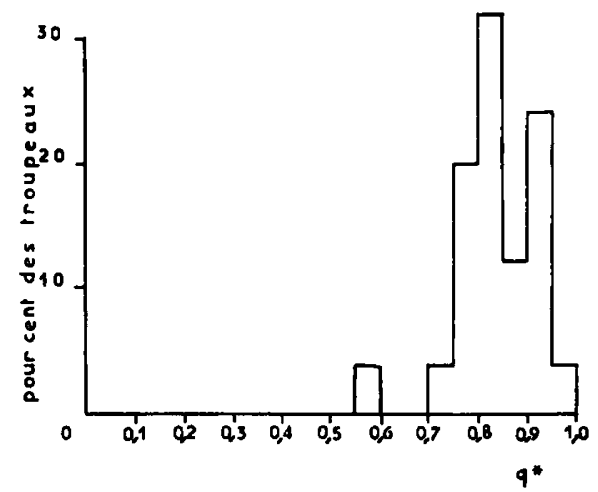

FIG. 3. - Histogramme monirant la répartition des 25 troupeaux privés du tableau 6 , selon la fréquence génétique de $\mathrm{w}$ chez les femelles $\left(\overrightarrow{\mathrm{q}}^{*}=0,847\right)$. Les troupeaux sont de iaille inégale variant de 50 ì 166 brebis, le nombre des mâles reproducteurs n'est pas connu.

troupeaux présentent un isolement géographique assez poussé. Or, dans l'élevage du mouton tel qu'il se pratique dans les troupeaux ci-dessus évoqués, qui appartiennent aux 200 troupeaux de sélection, les mâles, dont le nombre est réduit par rapport à celui des femelles (au plus I/20) ne sont généralement pas choisis dans le troupeau où ils sont employés et on les change assez souvent. Cependant, l'ampleur de la migration est considérablement freinée par la distance inais le fait d'observer dans des régions aussi éloignées qu'Olbia et Cagliari, des fréquences géniques très voisines est déjà un argument en faveur de l'existence de cet équilibre génique au niveau de la population ovine de l'île.

Pour expliquer un tel équilibre, il suffirait alors d'invoquer l'overdominance qui est une possibilité déduite de l'examen de nos données tant pour la production laitière que pour la prolificité des femelles. Dans le cas où cette overdominance se limiterait aux femelles, on aurait $W w>w w>W W$.

Bien entendu, tout cela reste à vérifier, ce qu'il nous est impossible de faire à partir des présentes données recueillies globalement comme sous-produits d'autres expérimentations.

Il semble possible en premier lieu de comparer les génotypes $W W$ et $W w$ 
puisqu'on pourra distinguer un certain nombre de brebis $W w$ (celles qui donnent un agneau sans pendeloques, ww).

En second lieu, on pourra s'attacher à préciser le phénomène de migration chez les mâles en faisant tout d'abord porter les efforts sur le sous-groupe des troupeaux de sélection qui échangent entre eux des reproducteurs sans en recevoir de l'immense majorité des troupeaux tout-venant auxquels cependant ils en fournissent. Une étude systématique de 1'origine des mâles employés et de leur durée d'utilisation devrait permettre de préciser l'ampleur du phénomène de migration. Par ailleurs, des preuves de l'existence de l'équilibre génique pourront être recherchées en précisant la durée depuis laquelle le phénomène de sélection génotypique est à l'œuvre parmi ces troupeaux de sélection.

\section{CONCLUSION}

Engagé comme une étude de routine annexe au travail de sélection de l'Istituto Zootecnico e caseario per la Sardegna, le présent travail dévoile un certain nombre d'aspects fort intéressants.

Pratiquement, tout d'abord, la connaissance du déterminisme exact du caractère présence de pendeloques peut, dans certains cas, aider à la reconnaissance de paternité ou de maternité, ce qui n'est pas toujours inutile.

En second lieu, on entrevoit un phénomène d'overdominance qui n'a sans doute pas été sans perturber déjà l'efficacité d'une sélection jusqu'à présent uniquement quantitative. Nul doute qu'à 1'avenir on doive en tenir compte dans les plans de sélection.

En troisième lieu, est soulevée l'intéressante question de la distribution stationnaire des fréquences géniques à travers une population très nombreuse divisée en de multiples unités.

$$
\text { Reģu pour publication en mai } 1970 .
$$

\section{SUMMARY}

\section{THE INHERITANCE OF WATTLES IN THE " SARDINIAN" MILK-EWE BREED}

In the Sardinian milk-ewe breed of the island of Sardinia, the existance of two or sometimes one wattle has been observed in a number of animals belonging to both sexes. A certain variability in the size and form of these appendices is noted. As is the case in goat populations and also other sheep breeds, geographically distant from Sardinia (the Valaque and Navajo ewes), the character responsible for the presence of wattles appears to be determined by a single dominant and autosomal gene $(w)$ with total or sub-total penetrance. This gene could have an influence on the reproduction and milk yield of the females; it was, however, not possible to study the males. The ewes with wattles ( $W W$ or $W w$ or both) appear to be superior for these two economically important characteristics, to the animals without wattles $w w$.

The gene frequency study of $w$ in 25 selection flocks $\left(\bar{q}^{*}=0.847\right)$ does not weaken the hypothesis of gene equilibrium. In this case, one should suppose the existance of overdominance, the $W w$ genotype being, at least in the females, superior to the two homozygotes. Direct comparative studies are necessary to clarify this point and it will also be necessary to undertake a detailed analysis of the migratory phenomenon of the males from one flock to another. It is also important to investigate the period and intensity of selection which might have acted in favour of one or another genotype so that an indication can be obtained on the existance or nonexistance of gene equilibrium on the level of the island's total population. 


\section{RÉFÉRENCES BIBLIOGRAPHIQUES}

Blanc L., I 897. Les pendeloques et le canal du Soyon. J. Anat. Physiol., Paris, 33, 283-302.

BlunN C. T., I943. Characteristics and production of old-type Navajo. J. Hered., 34, I41-I52.

CotTerman C. W., I964. Estimation of gene frequencies in nonexperimental populations. In KEMPTHORNE et al., Statistics and Mathematics in Biology, 449-465, Iowa State College Press, Ames, Iowa.

Goubaux A., I 852 . Note sur des corps qui sont appendus à l'extrémité supérieure du cou des chèvres et des moutons. Recl. méd. vét. prat., 335-342.

LUSH J. I., 1926. Inheritance of horns, wattles and colour in grade Toggenburg Goats. J. Hered., 17, 73-9x.

MouQUet M., 1895. Sur les pendeloques. Bull. Soc. centr. Méd. Vét., 49, 241-244.

Ricordeau G., I966. Hérédité des pendeloques en race Saanen. Différence de fécondité entre les génotypes avec et sans pendeloques. Ann. Zootech., 16, 263- 270.

SERra J. A., I948. Génétique du mouton. Mise au point critique. Publcóes Jte nac. Prod. pecúar. (ser. A), $\mathrm{n}^{\mathbf{0}} \mathbf{I}$.

VASIN B., 1928. Génétique du mouton. I. Hérédité de la couleur et des dessins (russe, rés. all.). Trav. Sta. Contr. Génét. anim. domest. Commissariat du Peuple à l'agriculture, Moscou (3). 